\title{
Near-Capacity Iterative Decoding of Binary Self-Concatenated Codes Using Soft Decision Demapping and 3-D EXIT Charts
}

\author{
Muhammad Fasih Uddin Butt, Student Member, IEEE, Raja Ali Riaz, Soon Xin Ng, Senior Member, IEEE, \\ and Lajos Hanzo, Fellow, IEEE
}

\begin{abstract}
In this paper 3-D Extrinsic Information Transfer (EXIT) charts are used to design binary Self-Concatenated Convolutional Codes employing Iterative Decoding (SECCC-ID), exchanging extrinsic information with the soft-decision demapper to approach the channel capacity. Recursive Systematic Convolutional (RSC) codes are selected as constituent codes, an interleaver is used for randomising the extrinsic information exchange of the constituent codes, while a puncturer helps to increase the achievable bandwidth efficiency. The convergence behaviour of the decoder is analysed with the aid of bit-based 3-D EXIT charts, for accurately calculating the operating $E_{b} / N_{0}$ threshold, especially when SP based soft demapper is employed. Finally, we propose an attractive system configuration, which is capable of operating within about $1 \mathrm{~dB}$ from the channel capacity.
\end{abstract}

Index Terms-Near-capacity code design, self-concatenated convolutional codes, SECCC, 3-D EXIT charts, iterative decoding.

\section{INTRODUCTION}

$\mathbf{T}$ HE philosophy of concatenated coding schemes was proposed by Forney in [1]. Turbo codes, which were developed in [2] constitute a class of error correction codes (ECC) based on parallel concatenated convolutional codes (PCCC) of two or more constituent codes. They are highperformance codes capable of operating near the Shannon limit [3]. Since their invention they have found diverse applications in bandwidth-limited communication systems, where the maximum achievable information rate has to be supported in the presence of transmission errors due to both Additive White Gaussian Noise (AWGN) and channel fading. Various bandwidth efficient turbo codes were proposed in [4], [5] and [6]. Serially concatenated convolutional codes (SCCC) [7]

Manuscript received October 1, 2008; revised April 20, 2009 and November 27, 2009; accepted February 17, 2010. The associate editor coordinating the review of this paper and approving it for publication was A. Gulliver.

M. F. U. Butt and R. A. Riaz are with the School of Electronics and Computer Science, University of Southampton, SO17 1BJ, Southampton, U.K., and also with the Department of Electrical Engineering, COMSATS Institute of Information Technology (CIIT), Islamabad 44000, Pakistan (email: \{mfub06r, rar06r\}@ecs.soton.ac.uk).

S. X. Ng and L. Hanzo are with the School of Electronics and Computer Science, University of Southampton, SO17 1BJ, Southampton, U.K. (e-mail: \{sxn, lh\}@ecs.soton.ac.uk).

The financial support of CIIT under the auspices of Higher Education Commission, Pakistan, and that of the EPSRC UK, as well as of the EU Optimix Project, is gratefully acknowledged.

The authors would like to thank the anonymous reviewers for their valuable comments, which greatly improved the presentation of the paper.

Digital Object Identifier 10.1109/TWC.2010.05.081254 have been shown to yield a performance comparable, and in some cases superior, to turbo codes. Iteratively-Decoded SelfConcatenated Convolutional Codes (SECCC-ID) proposed by Benedetto et al. [8] and Loeliger [9] constitute another attractive family of iterative detection aided schemes.

The concept of Extrinsic Information Transfer (EXIT) charts was proposed in [10] as a tool for analysing the convergence behaviour of iteratively decoded systems. EXIT charts constitute an efficient tool created to analyse each component of an iterative system independently.

SECCC is a low-complexity scheme involving only a single encoder and a single decoder. An EXIT chart based analysis of the iterative decoder provides an insight into its decoding convergence behaviour and hence it is helpful for finding the best coding schemes for creating SECCCs. An attractive approach to design Repeat-Accumulate (RA) codes using iterative detection and decoding using EXIT charts has been proposed in [11]. The RA code is a serial-concatenated coding scheme where a repetition code is employed as the outer code and a bit-by-bit accumulator is employed as the inner encoder. By contrast the SECCC scheme can be viewed as a parallel-concatenated coding scheme employing an odd-even separated turbo interleaver as discovered in [12]. Hence, the SECCC scheme is not a RA code. Similar to Bit-Interleaved Coded Modulation using the Iterative Decoding (BICM-ID) concept [13], we also employ iterations between the SECCC and the Soft Demapper in our SECCC-ID scheme. However, instead of using $N$ parallel bit interleavers as in BICM-ID, we only have one bit interleaver in our system. Note that the optimized mapping of [14] and the multidimensional mapping of [15] can also be employed for the SECCC-ID scheme. Furthermore, SECCC-ID codes have not been characterised in the literature in terms of their decoding convergence.

An SECCC-ID scheme was designed using Trellis Coded Modulation (TCM) as constituent codes with the aid of EXIT charts in [16]. The design proposed in [16] was symbol-based, therefore it had the inherent problem of exhibiting a mismatch between the EXIT curve and the bit-by-bit decoding trajectory. The main reason for the mismatch was that the EXIT charts were generated based on the assumption that the extrinsic information and the systematic information part of each TCM encoded symbol are independent of each other, which had a limited validity, since both the systematic and the parity bits were transmitted together as a single $2^{n+1}$-ary symbol. 


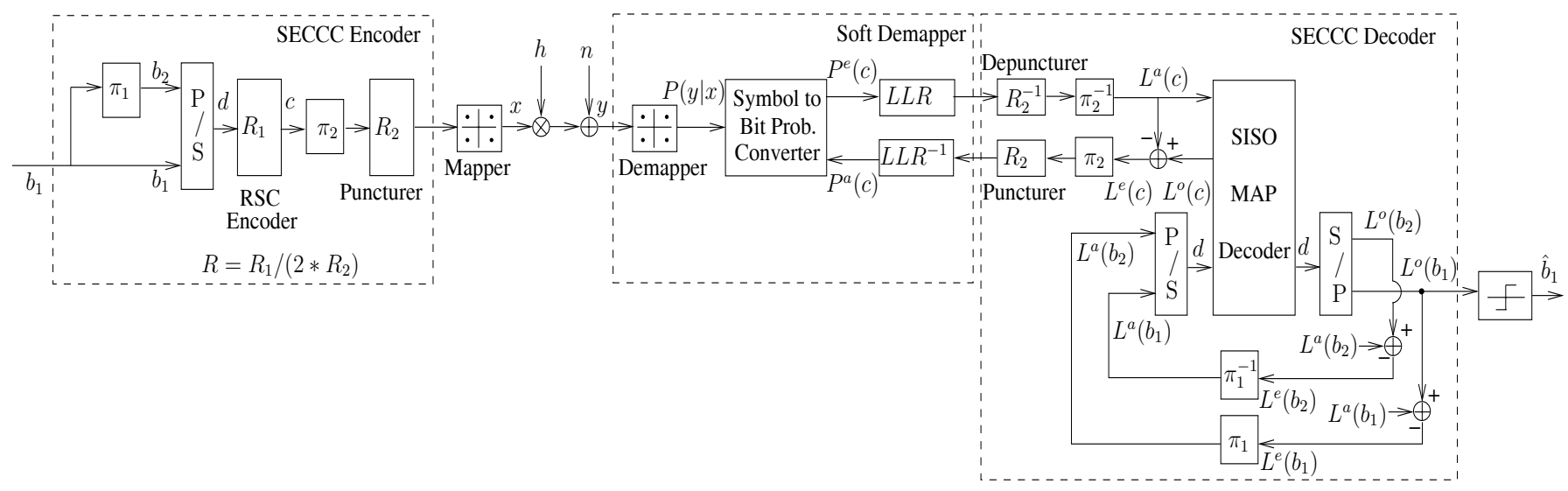

Fig. 1. SECCC-ID system exchanging soft information with the demapper.

More explicitly, the coded bits in each coded symbol are correlated $[17,18]$, hence they cannot convey the maximum possible information, which is equivalent to an entropy- or capacity-loss. Nonetheless, we found that the EXIT charts of the symbol-based SECCC scheme can be beneficially used, since the actual EXIT chart tunnel is always wider than the predicted EXIT chart tunnel [19]. Hence, the analysis was still valid, since it assisted us in finding the convergence SNR. The scheme proposed in [20] employs binary Recursive Systematic Convolutional (RSC) codes as constituent codes to eliminate the mismatch between the EXIT curves and the decoding trajectory inherited by the symbol-based TCM design by proposing a bit-based SECCC-ID design in order to create flexible SECCC schemes. It was suggested in [21] that a symbol-based scheme always has a lower convergence threshold compared to an equivalent binary scheme. In order to recover this information loss owing to employing binary rather than non-binary schemes, we will demonstrate that soft decision feedback is required between the SISO MAP decoder and the soft demapper [22].

Against this background, the novel contribution of this paper is the analysis of bit-based SECCC-ID codes and the design of flexible SECCC schemes that are capable of near-capacity operation over both AWGN and uncorrelated Rayleigh fading channels. Again we will demonstrate that in order to recover the 'bit-correlation-induced' entropy- or capacity-reduction owing to employing binary schemes, soft decision feedback is required between the SISO MAP decoder and the soft demapper. Two-stage iterative receivers can be analysed using 2-D EXIT charts, while their three-stage counterparts require 3-D EXIT charts, which were proposed in [23] and further studied in [24-26]. We will show that 3-D EXIT charts provide a unique insight into the design of near-capacity SECCC-ID codes. To elaborate a little further, in conventional 2-D EXIT charts a new EXIT curve is generated for each new channel SNR or channel model, while our 3-D EXIT chart representation of the SECCC component is channel-independent and hence has to be computed only once, regardless of the channel SNR. Hence we characterise each SECCC component code using two 3-D EXIT charts, which can then be used for designing an iterative decoding aided system employing any demapper type. Extrinsic information is exchanged across three concatenated decoder stages and the achievable decoding convergence is studied using 3-D EXIT charts. Finally, it is demonstrated that the best SECCC schemes perform within about $1 \mathrm{~dB}$ of both the AWGN and Rayleigh fading channels' capacity.

The organisation of the paper is as follows. After presenting our system model in Section II, we discuss the decoding convergence of the SECCC-ID scheme in Section III. Our results are discussed in Section IV and our conclusions are offered in Section V.

\section{SySTEM MOdEL}

We consider a rate $R=1 / 2$ SECCC scheme as an example to highlight the various system concepts considered in this paper. In all these examples we use both Set-Partitioning (SP) and Gray-coded Quadrature Phase-Shift Keying (QPSK) modulation. Both the AWGN and uncorrelated Rayleigh fading channels are considered. The notations $P($.$) and L($.$) in Fig. 1$ denote the logarithmic-domain symbol probabilities and the Logarithmic-Likelihood Ratio (LLR) of the bit probabilities, respectively. The notations $b$ and $c$ in the round brackets (.) in Fig. 1 denote information bits and coded bits, respectively. The specific nature of the probabilities and LLRs is represented by the subscripts $a$, $o$ and $e$, which denote in Fig. 1, a priori, a posteriori and extrinsic information, respectively.

As shown in Fig. 1, the input bit sequence $\left\{b_{1}\right\}$ of the selfconcatenated encoder is interleaved, yielding the bit sequence $\left\{b_{2}\right\}$. The resultant bit sequences are parallel-to-serial converted and then fed to the RSC encoder using the generator polynomials $\left(G_{r}=13, G_{1}=15, G_{2}=17\right)_{8}$ expressed in octal format and having a rate of $R_{1}=\frac{1}{3}$ and memory $\nu=3$, where $G_{r}$ specifies the feedback polynomial. Hence for every bit input to the SECCC encoder there are six output bits of the RSC encoder. At the output of the encoder there is an interleaver and then a rate $R_{2}=\frac{1}{3}$ puncturer, which punctures (does not transmit) two bits out of three encoded bits ${ }^{1}$. Hence, the overall code rate, $\mathrm{R}$ can be derived based on [27] as:

$$
R=\frac{R_{1}}{2 \times R_{2}}=\frac{1}{2}\left(\frac{1}{3\left(\frac{1}{3}\right)}\right)=\frac{1}{2},
$$

\footnotetext{
${ }^{1} R_{2}=\frac{x}{y}$ is the puncturing rate, where $(y-x)$ bit(s) are punctured for every $y$-bit segment. The interleaver $\pi_{2}$ is before the puncturer in Fig. 1, therefore the bits being punctured could be either the parity or the systematic bit.
} 
Therefore, at the output of the puncturer the number of encoded bits reduces from six to two bits, namely $\left(c_{1} c_{0}\right)$. Puncturing is used in order to increase the achievable bandwidth efficiency $\eta$. It can be observed that different codes can be designed by changing $R_{1}$ and $R_{2}$. These bits are then mapped to a QPSK symbol as $x=\mu\left(c_{1} c_{0}\right)$, where $\mu($. is the Gray-coded mapping function. Hence the bandwidth efficiency is given by $\eta=R \times \log _{2}(4)=1 \mathrm{bit} / \mathrm{s} / \mathrm{Hz}$ assuming a zero Nyquist roll-off-factor. The QPSK symbol $x$ is then transmitted over the communication channel. At the receiver side the received symbol is given by:

$$
y=h x+n,
$$

where $h$ is the channel's non-dispersive fading coefficient and $n$ is the AWGN having a variance of $N_{0} / 2$ per dimension. This signal is then used by the demapper for calculating the conditional probability density function (PDF) of receiving $y$, when a complex-value $x^{m}$ was transmitted:

$$
P\left(y \mid x=x^{m}\right)=\frac{1}{\pi N_{0}} \exp \left(-\frac{\left|y-h x^{m}\right|^{2}}{N_{0}}\right),
$$

where $x^{m}=\mu\left(c_{1} c_{0}\right)$ is the hypothetically transmitted QPSK symbol for $m \in\{0,1,2,3\}$. These PDFs are then passed through the Symbol to Bit Probability Converter of Fig. 1, which performs the following three steps:

1) Calculating the a posteriori probability of the transmitted signal

$$
P^{o}(x \mid y)=\frac{P(y \mid x) P(x)}{P(y)},
$$

where $P(y)$ is a constant term and can be ignored. For a symbol based system the probability of the transmitted signal $P(x)$ can be assumed to be a constant value. However, in a bit based system iterative decoding exchanging extrinsic information between the demodulator and decoder can be invoked based on $P(x)$.

2) From Bayes' rule and assuming that bits $c_{1}$ and $c_{0}$ are independent, we have

$$
P(x)=P\left(c_{1} c_{0}\right)=P\left(c_{1} \mid c_{0}\right) P\left(c_{0}\right)=P\left(c_{1}\right) P\left(c_{0}\right) .
$$

Therefore,

$$
P^{o}(x \mid y) \approx P(y \mid x) P\left(c_{1}\right) P\left(c_{0}\right) .
$$

3) The symbol probabilities are then converted to bit probabilities. The a posteriori probability of the coded bit $c_{i}=b$ is given by

$$
P^{o}\left(c_{i}=b \mid y\right)=\sum_{x \in \chi(i, b)}\left(P(y \mid x) \prod^{\operatorname{all}_{j}} P\left(c_{j}\right)\right),
$$

where $\chi(i, b)=\left\{\mu\left(c_{1} c_{0}\right), \forall c_{1}, c_{0} \in\{0,1\} \mid c_{i}=b\right\}$, which contains two phasor combinations of the QPSK modulated signal $x$. The bit index is specified by $i \in\{0,1\}$ and the value of the bit by $b \in\{0,1\}$. For higher order modulations the bits required are $m=\log _{2}(M)$, where $M$ is the number

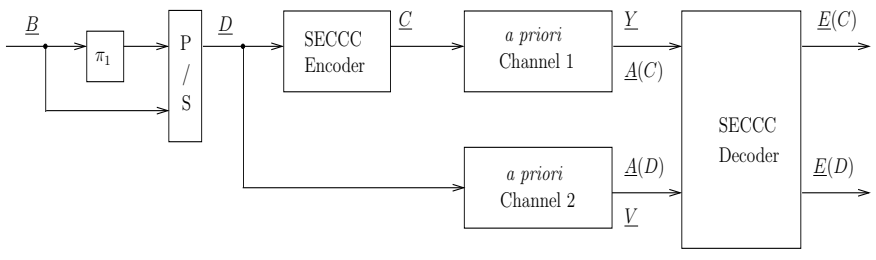

Fig. 2. Decoding model for an SECCC-ID scheme [16].

of constellation points. The extrinsic probability of $c_{i}$ is then calculated as

$$
P^{e}\left(c_{i}=b \mid y\right)=\sum_{x \in \chi(i, b)}\left(P(y \mid x) \prod_{j \neq i}^{\text {all }_{j}} P\left(c_{j}\right)\right) .
$$

The extrinsic bit probabilities are then converted to the corresponding bit-based LLRs by the block denoted as $L L R$ in Fig. 1, which are then passed through a soft depuncturer inserting zero LLRs at the punctured bit positions. The LLRs are then deinterleaved and fed to the Soft-Input SoftOutput (SISO) Maximum A Posteriori Probability (MAP) decoder [28]. The decoder of Fig. 1 is a self-concatenated decoder. It first calculates the extrinsic LLRs of the information bits, namely $L^{e}\left(b_{1}\right)$ and $L^{e}\left(b_{2}\right)$. Then they are appropriately interleaved to yield the a priori LLRs of the information bits, namely $L^{a}\left(b_{1}\right)$ and $L^{a}\left(b_{2}\right)$, as shown in Fig. 1. Self-concatenated decoding proceeds, until a fixed number of iterations is reached. The extrinsic LLRs of the codeword denoted by $L^{e}(c)$ at the output of the SISO decoder are fed back to the Soft Demapper of Fig. 1, which are interleaved by $\pi_{2}$ and then punctured according to $R_{2}$. These are then converted to the a priori bit probabilities $P_{b}^{a}(c)$ by the block denoted as $L L R^{-1}$ in Fig. 1 , to be fed to the APP demapper, which first converts them to symbol probabilities and then provides the improved extrinsic LLR $L^{e}(c)$ of the codeword at its output, thus completing the outer iteration between the SISO decoder and Soft Demapper. Apart from having inner self-concatenated iterations in the outer SECCC decoder, a fixed number of outer iterations exchange extrinsic information between the decoder and soft-demapper to yield the decoded bits $\hat{b}_{1}$.

\section{Decoding Convergence Analysis of SECCC-ID}

EXIT charts constitute powerful tools designed for analysing the convergence behaviour of concatenated codes without time-consuming bit-by-bit simulation of the actual system. They analyse the input/output mutual information characteristics of a SISO decoder by modelling the a priori LLRs either by an AWGN process or by its experimentally determined histogram and then computing the corresponding mutual information between the extrinsic LLRs as well as the corresponding bit-decisions. More explicitly, the employment of EXIT charts assumes having a sufficiently high interleaver length, so that the extrinsic LLRs can be assumed Gaussian distributed. The SNR value where the turbo-cliff [2] in the bit-error rate (BER) curve of a concatenated code appears can be successfully predicted with the aid of EXIT charts. 
The decoding model of the SECCC-ID scheme is portrayed in Fig. 2. It corresponds to either one of the hypothetical component decoders. Random variables (r.v.s.) are denoted with capital letters and their corresponding realizations with lower case letters. Sequences of random variables are indicated by underlining them. The information bit sequence is $\underline{B}$, which is interleaved and then parallel to serial converted. The resultant bits are denoted by $\underline{D}$, that are then encoded to yield the coded bit sequence $\underline{C}$ of Fig. 2, and transmitted over the communication channel often termed as the a priori channel 1 [29], which gives the a priori probabilities of the codeword $\underline{A}(C)$ as its output. The received symbol sequence is given by $\underline{Y}$, which is then fed to the SISO SECCC decoder. By contrast, the a priori channel 2 of Fig. 2 models the $a$ priori probabilities $\underline{A}(D)$ constituted by the combination of the information bits and their interleaved version referred to as the hypothetical dataword $\underline{D}$. The SECCC SISO decoder of Fig. 2 then computes the extrinsic bit probabilities revelant for both the codeword $\underline{E}(C)$ and the dataword $\underline{E}(D)$.

EXIT charts [10] visualize the input/output characteristics of the constituent SECCC decoder in terms of the average mutual information transfer. In the context of the SECCC decoder of Fig. 2, the EXIT chart visualises the following mutual information exchange:

1) average mutual information of $\underline{D}$ and $\underline{A}(D)$ :

$$
I_{A}(D)=\frac{1}{N_{D}} \sum_{k=1}^{N_{D}} I\left[D_{k} ; A\left(D_{k}\right)\right]
$$

2) average mutual information of $\underline{C}$ and $\underline{A}(C)$ :

$$
I_{A}(C)=\frac{1}{N_{C}} \sum_{k=1}^{N_{C}} I\left[C_{k} ; A\left(C_{k}\right)\right] ;
$$

3) average mutual information of $\underline{D}$ and $\underline{E}(D)$ :

$$
I_{E}(D)=\frac{1}{N_{D}} \sum_{k=1}^{N_{D}} I\left[D_{k} ; E\left(D_{k}\right)\right]
$$

4) average mutual information of $\underline{C}$ and $\underline{E}(C)$ :

$$
I_{E}(C)=\frac{1}{N_{C}} \sum_{k=1}^{N_{C}} I\left[C_{k} ; E\left(C_{k}\right)\right]
$$

where the number of symbols in the sequences $\underline{D}$ and $\underline{C}$ are given by $N_{D}$ and $N_{C}$, respectively. As depicted in Fig. 3, component 1 and 2 of SECCC decoders are associated with four mutual information transfers according to Eqs. (9)-(12). Hence two three-dimensional EXIT charts [23] are required for visualising the mutual information transfer between the hypothetical SECCC component decoders (namely for portraying each of the two outputs as a function of two inputs) and the EXIT curve of the combined SECCC decoder and the soft demapper (a two input, single output block).

Provided that a MAP decoder is used, the average extrinsic mutual information of $\underline{D}$ and $\underline{A}(D)$ may be computed from

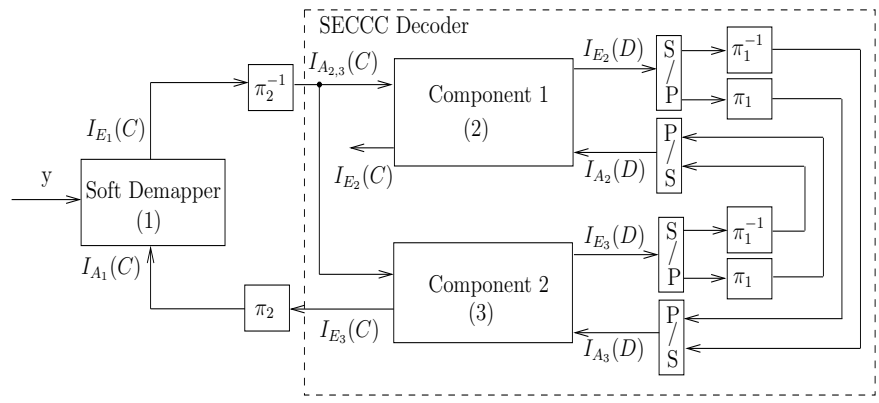

Fig. 3. Mutual information exchange between the three components of an SECCC-ID scheme [30].

Eqs. (10) and (11) of [17] as follows:

$$
\begin{aligned}
I_{E}(D)= & \frac{1}{N_{D}} \sum_{k=1}^{N_{D}} H\left(D_{k}\right)-H\left(D_{k} \mid E\left(D_{k}\right)\right) \\
= & \log _{2}\left(\mathcal{M}_{D}\right)- \\
& \frac{1}{N_{D}} \sum_{k=1}^{N_{D}} \mathrm{E}\left[\sum_{m=1}^{\mathcal{M}_{D}} E\left(D_{k}^{(m)}\right) \log _{2}\left(E\left(D_{k}^{(m)}\right)\right)\right],
\end{aligned}
$$

where $E\left(D_{k}^{(m)}\right)=P\left(D_{k}^{(m)} \mid \underline{Y}, \underline{V}_{[k]}\right)$ is the extrinsic probability of the hypothesized transmitted symbol $D_{k}^{(m)}$, for $m \in\left\{1, \ldots, \mathcal{M}_{D}\right\}$, which is provided by the MAP decoder. Here $\mathcal{M}_{D}=2^{L_{D}}$ and $L_{D}$ is the number of information bits ${ }^{2}$ in the SECCC input symbol of Fig. 2. Notice that the first expectation 'E' over the $N_{D}$ symbols in Eq. (13) may be removed, when $N_{D}$ is sufficiently large, yielding:

$I_{E}(D)=\log _{2}\left(\mathcal{M}_{D}\right)-\frac{1}{N_{D}} \sum_{k=1}^{N_{D}} \sum_{m=1}^{\mathcal{M}_{D}} E\left(D_{k}^{(m)}\right) \log _{2}\left[E\left(D_{k}^{(m)}\right)\right]$.

Similarly, we have [17]:

$I_{E}(C)=\log _{2}\left(\mathcal{M}_{C}\right)-\frac{1}{N_{C}} \sum_{k=1}^{N_{C}} \sum_{m=1}^{\mathcal{M}_{C}} E\left(C_{k}^{(m)}\right) \log _{2}\left[E\left(C_{k}^{(m)}\right)\right]$,

where $E\left(C_{k}^{(m)}\right)=P\left(C_{k}^{(m)} \mid \underline{Y}_{[k]}, \underline{V}\right)$ is the extrinsic probability of the hypothesized transmitted symbol $C_{k}^{(m)}$, for $m \in\left\{1, \ldots, \mathcal{M}_{C}\right\}$, generated by the MAP decoder and $N_{C}$ is assumed to be sufficiently large. Here, $\mathcal{M}_{C}=2^{L_{C}}$, where $L_{C}$ is the number of coded bits in the SECCC symbol.

The average a priori mutual information of both $\underline{D}$ and $\underline{C}$ may be modelled using the following assumptions $[17,31]$ :

1) the LLRs of the bits are Gaussian distributed: the LLR of an information or parity bit $s$, which can be either from the sequence $\underline{D}$ or $\underline{C}$, is given by [32]:

$$
z=h_{A} s+n_{A},
$$

where the variance of the AWGN $n_{A}$ is $\sigma_{A}^{2}$ per dimension and the equivalent 'fading factor' is given by $h_{A}=\sigma_{A}^{2} / 2$ [32];

\footnotetext{
${ }^{2}$ For binary EXIT chart, $L_{D}=1$
} 
2) the bits in a symbol are assumed to be independent of each other and uniformly distributed: the average a priori mutual information of a symbol sequence $\underline{D}$ (or $\underline{C}$ ), where each symbol $D_{k}$ (or $C_{k}$ ) consists of $L_{D}$ (or $L_{C}$ ) bits, is $L_{D}$ (or $L_{C}$ ) times the average a priori mutual information of a bit in the symbol.

The average a priori mutual information of a certain bit denoted as $s \in\left\{s^{(1)}=+1, s^{(2)}=-1\right\}$ and its LLR $z$ may be expressed as:

$$
I(S ; Z)=1-\frac{1}{2} \sum_{m=1}^{2} \mathrm{E}\left[\log _{2} \sum_{n=1}^{2} \exp \left(\Psi_{m, n}^{A}\right) \mid s^{(m)}\right],
$$

where we have $\exp \left(\Psi_{m, n}^{A}\right)=p\left(z \mid s^{(n)}\right) / p\left(z \mid s^{(m)}\right)$ and the conditional Gaussian PDF is given by:

$$
p(z \mid s)=\frac{1}{\sqrt{2 \pi \sigma_{A}^{2}}} \exp \left(\frac{\left(z-h_{A} s\right)^{2}}{2 \sigma_{A}^{2}}\right),
$$

while the exponent is given by:

$$
\Psi_{m, n}^{A}=\frac{-\left|\left(\sigma_{A}^{2} / 2\right)\left(s^{(m)}-s^{(n)}\right)+n_{A}\right|^{2}+\left|n_{A}\right|^{2}}{2 \sigma_{A}^{2}} .
$$

Note that another interpretation of Eq. (17) was given in [32, Eq. (14)]. We have a function $I_{A}=I(S ; Z)=J\left(\sigma_{A}\right)$, with $J\left(\sigma_{A}\right)$ being monotonically increasing and therefore invertible. Hence, at a given $I_{A}$ value we may find the corresponding $\sigma_{A}$ value from $J^{-1}\left(I_{A}\right)$. Finally, one may compute the corresponding LLR value $z$ from Eq. (16). The a priori mutual information of an $L_{D}$-bit symbol $D_{k}$ is given by:

$$
I\left(D_{k} ; Z_{(k)}\right)=\sum_{i=1}^{L_{D}} I\left[s_{(k, i)}^{D} ; z_{(k, i)}^{D}\right],
$$

where $z_{(k)}^{D}=\left\{z_{(k, 1)}^{D}, \ldots, z_{\left(k, L_{D}\right)}^{D}\right\}$ is the LLR sequence, which is related to the $L_{D}$ bits of $D_{k}$ and $z_{(k, l)}^{D}$ is the LLR of $s_{(k, l)}^{D}$, which is the $l$ th bit in the $k$ th symbol $D_{k}$.

The EXIT charts of self-concatenated codes are typically similar to those of the family of parallel concatenated codes [2, 17,18], where an open EXIT tunnel exists if the EXIT curve does not intersect with the line connecting the point $\left(I_{A}=0, I_{E}=0\right)$ to the point $\left(I_{A}=1, I_{E}=1\right)$ in the EXIT chart. In [16] EXIT charts were successfully used to compare the performance of non-binary SECCC schemes. This paper uses the same method by finding the threshold $E_{b} / N_{0}$ point by calculating the EXIT curve of the identical decoder components and then plotting them together in the EXIT chart, as shown in Fig. 7. The $E_{b} / N_{0}$ value, where the two EXIT curves touch each other is termed as the threshold $E_{b} / N_{0}$ point denoted by $\Lambda$, which is the point where the turbo-cliff [2] region starts and beyond which the EXIT tunnel becomes 'just' open. If uncorrelated extrinsic information is available, then all of the symbol-by-symbol decoding trajectories will reach the $\left(I_{A}, I_{E}\right)=(1,1)$ point [10] for $E_{b} / N_{0}$ greater than $\Lambda$. The various coding schemes considered in this paper are characterised in Table I. They are identified by the code rate $\left(R_{1}\right)$, puncturing rate $\left(R_{2}\right)$, the overall code rate $(R)$,

\begin{tabular}{|c|c|c|c|c|c|c|c|}
\hline \multirow[t]{2}{*}{$\begin{array}{l}\text { SECCC- } \\
\text { ID } \\
\text { Scheme }\end{array}$} & \multirow[t]{2}{*}{ Mapping } & \multirow[t]{2}{*}{$\nu$} & \multirow[t]{2}{*}{$\begin{array}{l}\eta \\
\text { (bit/s } \\
/ \mathrm{Hz} \text { ) }\end{array}$} & \multicolumn{2}{|c|}{$\begin{array}{l}\text { AWGN } \\
\text { Channel } \\
E_{b} / N_{0}(\mathrm{~dB})\end{array}$} & \multicolumn{2}{|c|}{$\begin{array}{l}\text { Rayleigh } \\
\text { Channel } \\
E_{b} / N_{0}(\mathrm{~dB})\end{array}$} \\
\hline & & & & $\Lambda$ & $\omega$ & $\Lambda$ & $\omega$ \\
\hline $\begin{array}{l}\mathrm{R}_{1}=1 / 2, \\
\mathrm{R}_{2}=3 / 4, \\
\mathrm{R}=1 / 3\end{array}$ & $\begin{array}{l}\text { GM } \\
\text { SP } \\
\text { GM } \\
\text { SP }\end{array}$ & 2 & 0.67 & $\begin{array}{l}0.71 \\
0.25 \\
0.44 \\
0.5\end{array}$ & -0.6 & $\begin{array}{l}1.81 \\
1.35 \\
1.56 \\
1.55\end{array}$ & 1 \\
\hline $\begin{array}{l}\mathrm{R}_{1}=1 / 2, \\
\mathrm{R}_{2}=1 / 2, \\
\mathrm{R}=1 / 2\end{array}$ & $\begin{array}{l}\text { GM } \\
\text { SP } \\
\text { GM } \\
\text { SP }\end{array}$ & 3 & 1 & $\begin{array}{l}1.45 \\
1.0 \\
1.2 \\
1.25\end{array}$ & 0.19 & $\begin{array}{l}3.4 \\
3.4 \\
3.2 \\
3.3\end{array}$ & 1.83 \\
\hline $\begin{array}{l}\mathrm{R}_{1}=1 / 2, \\
\mathrm{R}_{2}=1 / 3, \\
\mathrm{R}=3 / 4\end{array}$ & $\begin{array}{l}\text { GM } \\
\text { SP } \\
\text { GM } \\
\text { SP }\end{array}$ & 2 & 1.5 & $\begin{array}{l}3.44 \\
3.2 \\
3.24 \\
3.2\end{array}$ & 2 & $\begin{array}{l}8.54 \\
8.4 \\
8.09 \\
8.1\end{array}$ & 6 \\
\hline $\begin{array}{l}\mathrm{R}_{1}=1 / 3, \\
\mathrm{R}_{2}=2 / 3, \\
\mathrm{R}=1 / 4\end{array}$ & $\begin{array}{l}\text { GM } \\
\text { SP }\end{array}$ & 3 & 0.5 & $\begin{array}{l}0.17 \\
0.07\end{array}$ & -0.8 & $\begin{array}{l}0.96 \\
0.82\end{array}$ & -0.2 \\
\hline $\begin{array}{l}\mathrm{R}_{1}=1 / 3, \\
\mathrm{R}_{2}=1 / 3, \\
\mathrm{R}=1 / 2\end{array}$ & $\begin{array}{l}\text { GM } \\
\text { SP }\end{array}$ & 3 & 1 & $\begin{array}{l}1.28 \\
1.23\end{array}$ & 0.19 & $\begin{array}{l}3.3 \\
3.4\end{array}$ & 1.83 \\
\hline $\begin{array}{l}\mathrm{R}_{1}=1 / 3, \\
\mathrm{R}_{2}=1 / 4, \\
\mathrm{R}=2 / 3\end{array}$ & $\begin{array}{l}\text { GM } \\
\text { SP }\end{array}$ & 3 & 1.33 & $\begin{array}{l}2.43 \\
2.37\end{array}$ & 1.5 & $\begin{array}{l}5.95 \\
5.7\end{array}$ & 3 \\
\hline
\end{tabular}
code memory $\nu$ and bandwidth efficiency $\eta$, expressed in
TABLE I

VARIOUS SECCC-ID SCHEMES AND THEIR THRESHOLDS.

bit/s/Hz. Furthermore, $O$ denotes the number of outer iterations between the demapper and the decoder and $I$ denotes the number of inner self-concatenated iterations. In all the codes considered in Table I the thresholds are calculated for $O=40$ and $I=40$ for the SP and Gray mapping schemes, respectively. Finally, the channel capacity limit $\omega$ is also expressed in $\mathrm{dBs}$ [33], as tabulated in Table I. For $R_{1}=1 / 2$ and $\nu=2$, the generator polynomial $G=(7,5)_{8}$ is used, whereas for $\nu=3, G=(13,15)_{8}$ is employed. For $R_{1}=1 / 3$ and $\nu=3, G=(13,15,17)_{8}$ is used, where the first number in the generator polynomial represents the feedback polynomial. The EXIT charts recorded for the binary SECCC-ID schemes of Table I are shown in Figs. 4, 5, 6 and 7.

The mutual information exchange between the components of an SECCC-ID scheme is portrayed in Fig. 3, which shows the SECCC-ID decoder of Fig. 1 as two hypothetical component decoders. The hypothetical component 2 of the SECCC decoder of Fig. 3 receives inputs from and provides outputs for both the soft demapper and the hypothetical component 1 SECCC decoder of Fig. 3. Hence we have two EXIT surfaces in Fig. 4, the first one corresponding to the component 2 decoder's average mutual information $I_{E_{3}}(C)$ provided for the soft demapper, while the second one corresponding to $I_{E_{3}}(D)$ supplied for the component 1 SECCC decoder, as shown in Fig. 3. The same procedure can be used to calculate the two EXIT surfaces for the average mutual information of the component 1 decoder. One of the EXIT surfaces corresponds to the mutual information $I_{E_{2}}(C)$ provided for the soft demapper (not used) in Fig. 3. Similarly, the component 1 SECCC decoder has the other EXIT surface characterising its average mutual information $I_{E_{2}}(D)$ forwarded to the hypothetical component 2 SECCC decoder of Fig. 3. By contrast, the soft demapper has a single EXIT surface characterising its average mutual information $I_{E_{1}}(C)$ forwarded to component 1 and 2 of the SECCC decoder of Fig. 3. 


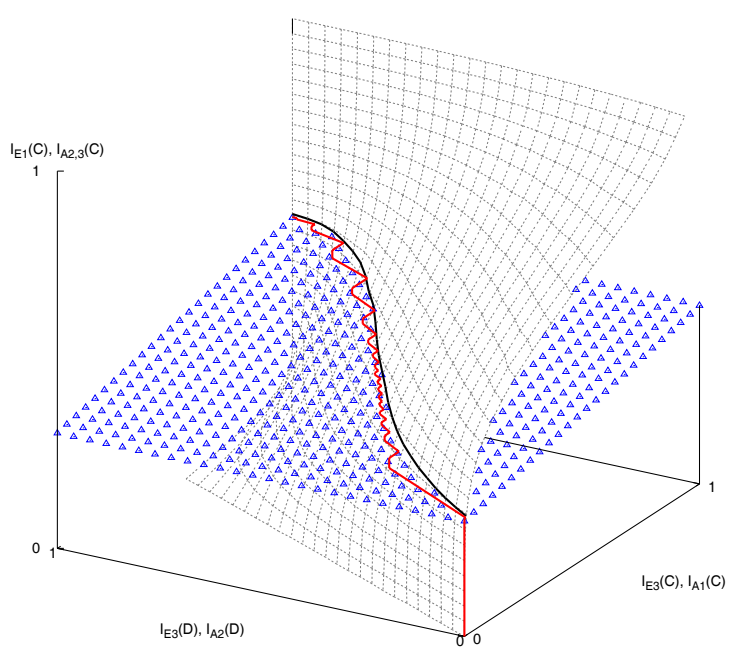

Fig. 4. 3-D EXIT surfaces of SECCC decoder (dotted) and Soft Demapper (triangles) along with a 'snap-shot' decoding trajectory for $R_{1}=1 / 2$ and $R_{2}=3 / 4$, QPSK-assisted SECCC-ID, $\nu=2, \eta=0.67 \mathrm{bit} / \mathrm{s} / \mathrm{Hz}$ at $E_{b} / N_{0}=$ $1.55 \mathrm{~dB}$ using SP mapping, for transmission over a Rayleigh fading channel.

The scheme using $R_{1}=1 / 2, R_{2}=3 / 4, \nu=2$ and employing the SP based Soft Demapper is shown in Figs. 4 and 5. Specifically, the EXIT surface marked with triangles in Fig. 4 was computed based on the Soft Demapper's output $I_{E_{1}}(C)$ at the given $I_{E_{3}}(D)$ value of the component 2 SECCC Decoder and $I_{A_{1}}(C)$ of the Soft Demapper's abscissa values. By contrast, the steeply rising EXIT surface drawn using dotted lines in Fig. 4 was computed based on the component 2 decoder's outputs $I_{E_{3}}(C)$ and $I_{E_{3}}(D)$ at the given $I_{A_{2,3}}(C)$ value. Note that the Soft Demapper characteristic is independent of $I_{E_{3}}(D)$ gleaned from the output of the component 2 decoder, as seen in Fig. 3. As we can see from Fig. 4, the decoding trajectory is computed at $E_{b} / N_{0}=1.55 \mathrm{~dB}^{3}$. The symbol-by-symbol decoding trajectory (solid line) is based on the average mutual information of the component 2 SECCC decoder's output, namely on $I_{E_{3}}(C)$, and it evolves within the space under the EXIT surface marked with triangles but above the EXIT surface drawn using dotted lines, which means that it matches the 3-D EXIT curves.

Similarly, the EXIT surface of Fig. 5 spanning from the horizontal line $\left[I_{A_{2}}(D)=\{0 \rightarrow 1\}, I_{E_{2}}(D)=0\right.$, $\left.I_{A_{2,3}}(C)=0\right]$ to the horizontal line $\left[I_{A_{2}}(D)=\{0 \rightarrow 1\}\right.$, $\left.I_{E_{2}}(D)=1, I_{A_{2,3}}(C)=1\right]$, represents the first hypothetical SECCC decoder component. Since in case of SECCCs these are identical components, we only have to compute the EXIT surface of a single component and the other is its mirror image [16]. The EXIT surfaces of the two hypothetical decoder components are plotted within the same EXIT chart together with their corresponding decoding trajectory for the sake of visualizing the exchange of extrinsic information between the decoders. The EXIT surfaces of the proposed scheme match

\footnotetext{
${ }^{3}$ Note that there is a small but still beneficial vertical step in the decoding trajectory (Figs. 4 and 5) after each iteration of the SECCC decoder and the Soft Demapper. This justifies the use of 3-D EXIT charts as compared to 2-D EXIT charts, where this gain cannot be observed.
}

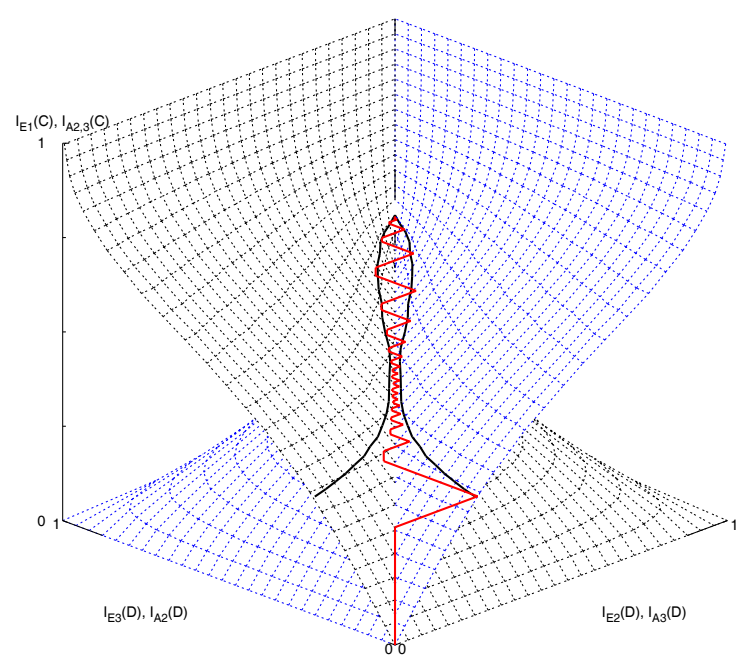

Fig. 5. 3-D EXIT surfaces of the two identical hypothetical SECCC decoder components and a 'snap-shot' decoding trajectory for $R_{1}=1 / 2$ and $R_{2}=3 / 4$, QPSK-assisted SECCC-ID, $\nu=2, \eta=0.67 \mathrm{bit} / \mathrm{s} / \mathrm{Hz}$ at $E_{b} / N_{0}=1.55 \mathrm{~dB}$ using SP mapping for transmission over a Rayleigh fading channel.

exactly the decoding trajectories computed from the bit-by-bit simulations.

The 3-D EXIT surfaces of the SECCC decoder and the two distinct decoding trajectories - one for SP (iterating solid line) and the other for Gray mapping (iterating bold solid line) based Soft Demapper - were recorded for the binary SECCC schemes operating closest to the Rayleigh channel's capacity, which are given in Fig. 6.

Each EXIT surface was calculated based on 10 transmission frames, each consisting of $24 \times 10^{3}$ information bits. Each Monte-Carlo-simulation based snapshot decoding trajectory was computed based on a block length of $120 \times 10^{3}$ information bits.

In Fig. 6, the scheme using $R_{1}=1 / 2, R_{2}=3 / 4, \nu=2$ and employing Gray mapping acquires an open EXIT tunnel at $E_{b} / N_{0}=1.9 \mathrm{~dB}$, when communicating over an uncorrelated Rayleigh fading channel. For this scheme the threshold $\Lambda$ is at $1.81 \mathrm{~dB}$ as recorded in Table $\mathrm{I}$, which is $0.81 \mathrm{~dB}$ away from the Rayleigh channel's capacity.

To calculate the threshold of a given SP mapping based SECCC-ID scheme, we have to rely on 3-D EXIT charts to analyse the mutual information exchange gain achieved, while iterating between the soft demapper and the decoder. This is shown in Fig. 4 and 5. The intersection of the surfaces in Fig. 4 represents the points of convergence between the SNRdependent soft demapper and the SNR-independent SECCCID decoder. At these intersection points we have shown a solid line. The corresponding $I_{E_{2}}(D)$ values associated with the curve of intersection of the surfaces in Fig. 4 and its mirror image are projected onto the surfaces seen in Fig. 5. Fig. 5 also shows the Monte-Carlo-simulation based decoding trajectory matching these EXIT curves. These EXIT curves are projected onto $I_{E_{1}}(C)=0$ for yielding Fig. 7(b). The 2D projection seen in Fig. 7(b) for the Rayleigh fading channel has a threshold of $1.35 \mathrm{~dB}$. Hence, an overall gain of $0.46 \mathrm{~dB}$ is attained compared to the Gray mapping performance seen in 


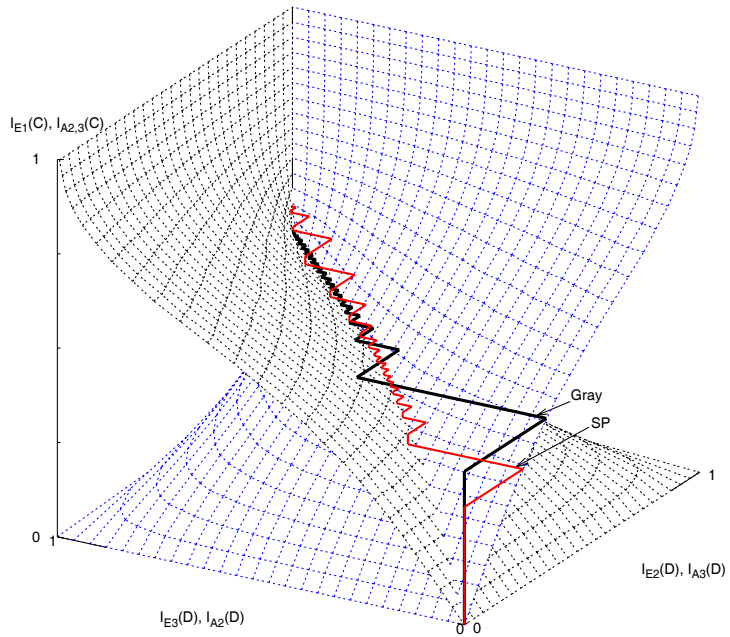

Fig. 6. EXIT chart and two 'snap-shot' decoding trajectories for $R_{1}=1 / 2$ and $R_{2}=3 / 4$, QPSK-assisted SECCC-ID, $\nu=2, \eta=0.67 \mathrm{bit} / \mathrm{s} / \mathrm{Hz}$ at $E_{b} / N_{0}=$ $1.55 \mathrm{~dB}$ using SP mapping and at $E_{b} / N_{0}=1.9 \mathrm{~dB}$ using Gray mapping, for transmission over a Rayleigh fading channel.

Fig. 7(a). The uncorrelated Rayleigh fading channel's capacity is $1 \mathrm{~dB}$ for this scheme, hence, it operates $0.35 \mathrm{~dB}$ away from capacity.

The 2-D EXIT curves recorded for the case of Rayleigh fading channel are shown in Fig. 7(a). These exemplify the method of finding thresholds for the Gray mapped SECCCID scheme using $\nu=2, R_{1}=1 / 2$ and $R_{2}=3 / 4$. 2-D EXIT curves have been used for the case of Gray mapping because there is no mutual information exchange gain between the soft demapper and the decoder. Hence, the threshold can be calculated using 2-D EXIT charts for the case of Gray mapping ${ }^{4}$. We found that when employing the $\nu=2$ RSC code, all SECCC schemes exhibited EXIT curves having similar trends to those in Fig. 7(a), where the tunnel at the top-right corner becomes very narrow. Hence, a higher SNR was required for the decoding trajectory to pass through the tunnel. As a result, their performance tends to be farther away from the channel capacity. The threshold of $E_{b} / N_{0}=1.81 \mathrm{~dB}$ is shown in Fig. 7(a) and in Table I, which is $0.81 \mathrm{~dB}$ away from the Rayleigh fading channel's capacity.

The interleaver $\pi_{1}$ of Fig. 1 is used in all of the schemes considered in Table I, which renders the information bits, more-or-less uncorrelated. This is a necessary requirement for the generation of accurate EXIT charts, because they require the LLRs of the information bits to be Gaussian distributed. The interleaver used after the RSC encoder of Fig. 1, namely $\pi_{2}$, randomises the coded bits before the puncturer.

\section{RESUlts And Discussions}

The EXIT charts discussed in Section III were used to find near-capacity SECCC-ID schemes for $\nu=\{2,3\}$, when communicating over AWGN and uncorrelated Rayleigh fading channels.

\footnotetext{
${ }^{4}$ Note that it is possible to simply use the 3-D EXIT chart of the SECCCID scheme when using the Gray mapper, without having to compute another 2-D EXIT curve for the SECCC scheme at a given SNR or channel model.
}

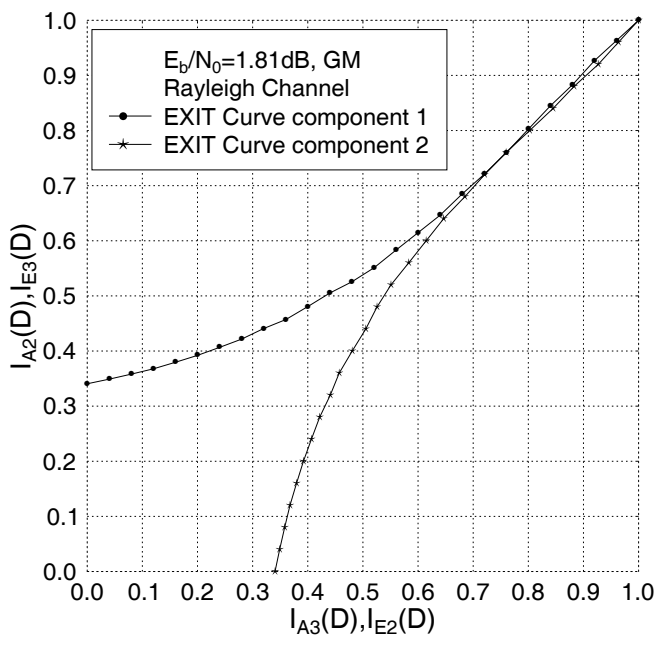

(a) Gray Mapping

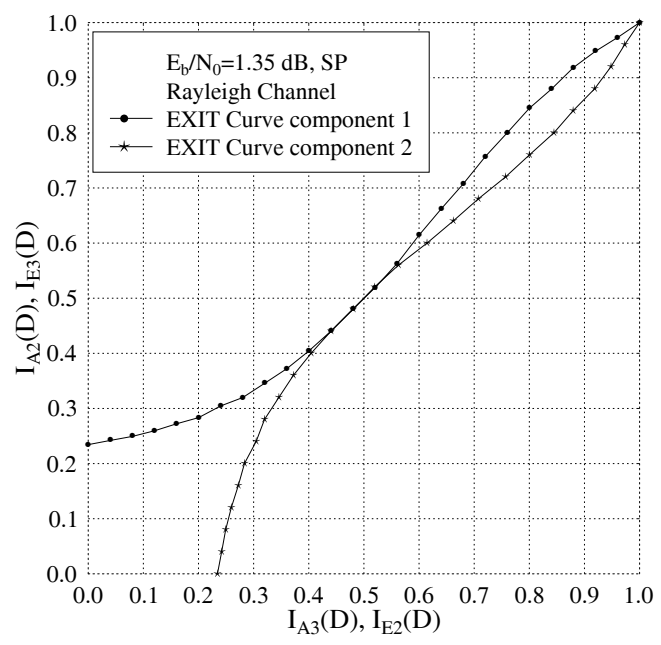

(b) 2D Projection of SP Mapping

Fig. 7. EXIT curves of the $R_{1}=1 / 2$ and $R_{2}=3 / 4, \nu=2$, SECCC-ID scheme to find the corresponding thresholds, operating over an uncorrelated Rayleigh fading channel.

The threshold predicted by the EXIT chart analysis detailed in Section III closely matches with the actual threshold observed in the BER curve given by the specified $E_{b} / N_{0}$ value, where there is a sudden drop of the BER after a certain number of decoding iterations, as shown in Figs. 8 and 9. Hence it becomes possible to attain an infinitesimally low BER beyond the threshold, provided that the block length is sufficiently long and the number of decoding iterations is sufficiently high. Again, the BER versus $E_{b} / N_{0}$ performance curves of the best performing QPSK-assisted SECCC-ID schemes having $R_{1}=1 / 2$ and $R_{2}=3 / 4$, recorded from our bit-by-bit simulations are shown in Figs. 8 and 9. We considered an information block length of $120 \times 10^{3}$ bits per frame, for $10^{3}$ frames and the number of decoding iterations $(I)$ are fixed to 40 . Figs. 8 and 9 show the $E_{b} / N_{0}$ difference between the channel capacity and the system operating at a BER of $10^{-3}$ marked by dotted lines, which was recorded for the bestperforming SECCC-ID scheme the code memory of $\nu=2$. The SP mapping scheme operates $0.47 \mathrm{~dB}$ away from capacity, 


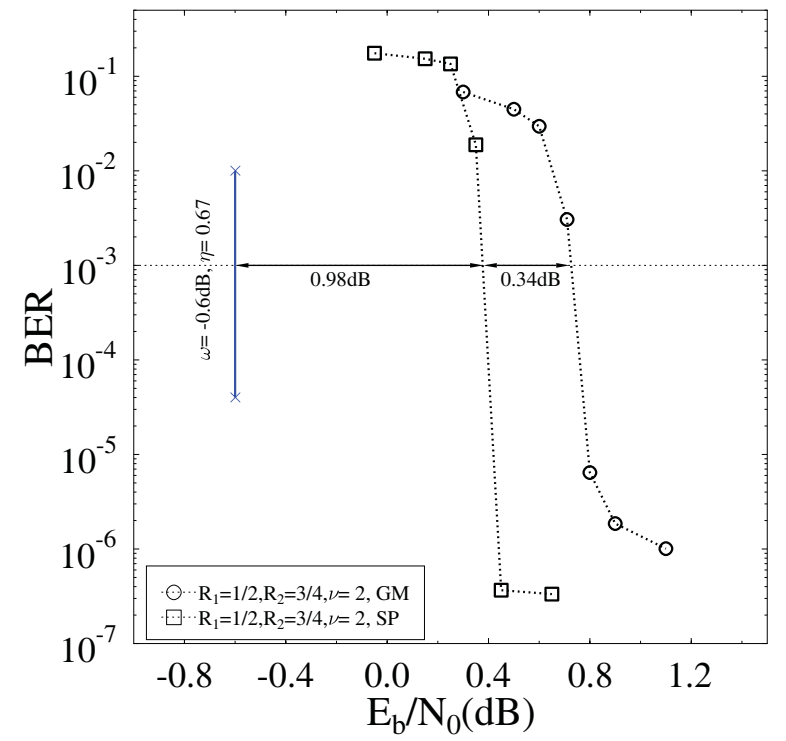

Fig. 8. The BER versus $E_{b} / N_{0}$ performance of Gray and SP mapped QPSK-assisted SECCC-ID schemes, $R_{1}=1 / 2, R_{2}=3 / 4$, and $I=40$ decoding iterations for $\nu=2$, operating over an AWGN channel.

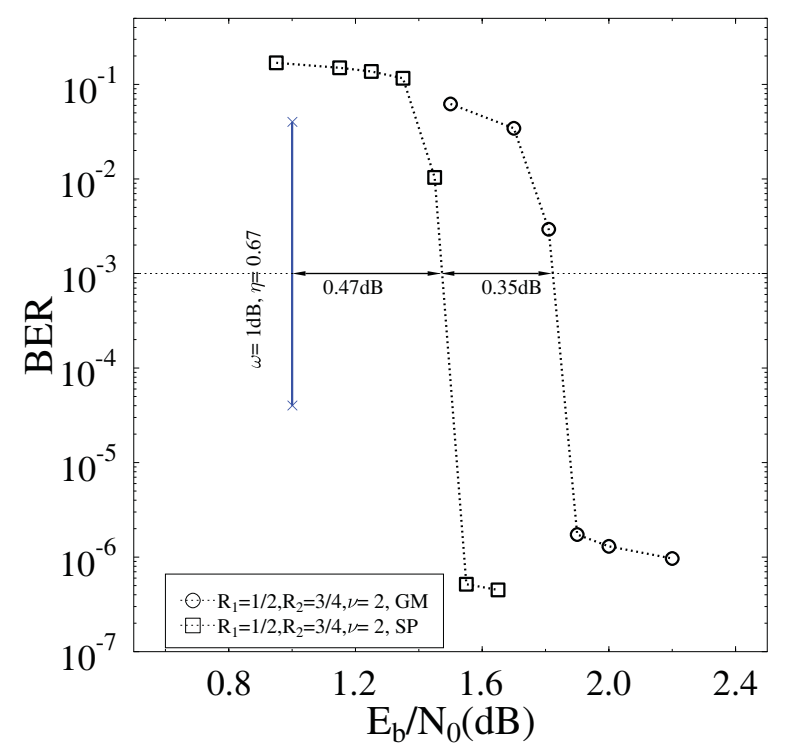

Fig. 9. The BER versus $E_{b} / N_{0}$ performance of Gray and SP mapped QPSK-assisted SECCC-ID schemes, $R_{1}=1 / 2, R_{2}=3 / 4$, and $I=40$ decoding iterations for $\nu=2$, operating over an uncorrelated Rayleigh fading channel.

which is $0.35 \mathrm{~dB}$ better compared to that of Gray mapping scheme at a BER of $10^{-3}$.

As we can see by studying Table I and Figs. 8 and 9, the BER thresholds are accurately predicted by the EXIT charts. Hence, the binary EXIT chart is useful for finding the best SECCC-ID schemes that are capable of decoding convergence at the lowest possible $E_{b} / N_{0}$ value. We apply the same method of calculating the BER thresholds for a range of SECCC-ID schemes, as detailed in Table I.

For the scheme employing $\nu=2, R_{1}=1 / 2$ and $R_{2}=3 / 4$, the distance from capacity is $(0.25+0.6)=0.85 \mathrm{~dB}$ and $(1.35-1)=0.35 \mathrm{~dB}$, when communicating over AWGN and Rayleigh fading channels, respectively. Another scheme, which performs close to capacity, employs $\nu=3, R_{1}=1 / 3$ and $R_{2}=2 / 3$, as shown in Table I. This scheme is capable of operating within $(0.07+0.8)=0.87 \mathrm{~dB}$ and $(0.82+0.2)=$ $1.02 \mathrm{~dB}$ from the capacity of the AWGN and Rayleigh fading channels, respectively, while employing the SP mapper.

\section{CONCLUSIONS}

We have designed near-capacity SECCC-ID schemes based on their decoding convergence analysis. The SECCC schemes invoke binary RSC codes and different puncturing rates. The puncturer is used to increase the achievable bandwidth efficiency. The interleaver placed before the puncturer helps randomise the puncturing pattern. Good SECCC parameters were found for assisting the SECCC-ID scheme in attaining decoding convergence at the lowest possible $E_{b} / N_{0}$ value, when communicating over both AWGN and uncorrelated Rayleigh fading channels. We have demonstrated that 3-D EXIT charts are useful for designing near-capacity SECCCID codes. Furthermore, 3-D EXIT charts may also be used to design a SECCC-ID scheme concatenated with an outer codec, such as a video codec for enabling soft information exchange between the SECCC-ID decoder and the video decoder. The SECCC-ID schemes designed are capable of operating within $1 \mathrm{~dB}$ of the AWGN as well as Rayleigh fading channel's capacity. Our future work will focus on designing SECCC schemes operating closer to capacity, while maintaining a high bandwidth efficiency. Furthermore, we will investigate the performance of such SECCC-ID schemes in non-coherently detected cooperative communication systems.

\section{REFERENCES}

[1] G. Forney, Concatenated Codes. MIT Press, Cambridge, MA,, 1966.

[2] C. Berrou, A. Glavieux, and P. Thitimajshima, "Near Shannon limit error correcting coding and decoding: turbo codes," in Proc. IEEE International Conference on Communications ICC '93, vol. 2, Geneva, Switzerland, pp. 1064-1070, May 1993.

[3] C. Shannon, "A mathematical theory of communication," Bell Syst. Tech. J., vol. 27, pp. 623-656, 1948.

[4] S. Le Goff, A. Glavieux, and C. Berrou, "Turbo-codes and high spectral efficiency modulation," in Proc. IEEE International Conference on Communications, New Orleans, LA, USA, pp. 645-649, May 1994.

[5] S. Benedetto, D. Divsalar, G. Montorsi, and F. Pollara, "Bandwidth efficient parallel concatenated coding schemes," Electron. Lett., vol. 31, pp. 2067-2069, Nov. 1995.

[6] P. Robertson and T. Wörz, "Coded modulation scheme employing turbo codes," Electron. Lett., vol. 31, pp. 1546-1547, Aug. 1995.

[7] S. Benedetto, D. Divsalar, G. Montorsi, and F. Pollara, "Serial concatenated trellis coded modulation with iterative decoding," in Proc. IEEE International Symposium on Information Theory, Ulm, p. 8, June/July 1997.

[8] S. Benedetto, D. Divsalar, G. Montorsi, and F. Pollara, "Selfconcatenated trellis coded modulation with self-iterative decoding," in Proc. IEEE Global Telecommunications Conference, vol. 1, Sydney, NSW, Australia, pp. 585-591, 1998.

[9] H. A. Loeliger, "New turbo-like codes," in Proc. IEEE International Symposium on Information Theory, Ulm, p. 109, June/July 1997.

[10] S. ten Brink, "Convergence of iterative decoding," Electron. Lett., vol. 35, pp. 806-808, May 1999.

[11] S. ten Brink and G. Kramer, "Design of repeat-accumulate codes for iterative detection and decoding," IEEE Trans. Signal Process., vol. 51, pp. 2764-2772, Nov. 2003.

[12] S. X. Ng, M. F. U. Butt, and L. Hanzo, "On the union bounds of selfconcatenated convolutional codes," IEEE Signal Process. Lett., vol. 16, pp. 754-757, Sept. 2009.

[13] X. Li, A. Chindapol, and J. A. Ritcey, "Bit-interleaved coded modulation with iterative decoding and 8PSK signaling," IEEE Trans. Commun., vol. 50, pp. 1250-1257, Aug. 2002. 
[14] F. Schreckenbach, N. Görtz, J. Hagenauer, and G. Bauch, "Optimization of symbol mappings for bit-interleaved coded modulation with iterative decoding," IEEE Commun. Lett., vol. 7, pp. 593-595, Dec. 2003.

[15] N. H. Tran, and H. H. Nguyen, "A Novel Multi-Dimensional Mapping of 8-PSK for BICM-ID," IEEE Trans. Wireless Commun., vol. 6, pp. 11331142, Mar. 2007.

[16] M. F. U. Butt, S. X. Ng, and L. Hanzo, "EXIT chart aided design of near-capacity self-concatenated trellis coded modulation using iterative decoding," in Proc. 67th IEEE Vehicular Technology Conference, VTC '08 Spring, Marina Bay, Singapore, pp. 734-738, May 2008.

[17] J. Kliewer, S. X. Ng, and L. Hanzo, "Efficient computation of EXIT functions for nonbinary iterative decoding," IEEE Trans. Commun., vol. 54, pp. 2133-2136, Dec. 2006.

[18] H. Chen and A. Haimovich, "EXIT charts for turbo trellis-coded modulation," IEEE Commun. Lett., vol. 8, pp. 668-670, Nov. 2004.

[19] S. X. Ng, O. R. Alamri, Y. Li, J. Kliewer, and L. Hanzo, "Near-capacity turbo trellis coded modulation design based on EXIT charts and union bounds," IEEE Trans. Commun., vol. 56, pp. 2030-2039, Dec. 2008.

[20] M. F. U. Butt, R. A. Riaz, S. X. Ng, and L. Hanzo, "Near-capacity iteratively decoded binary self-concatenated code design using EXIT charts," in Proc. IEEE Global Communications Conference, GLOBECOM '08, New Orleans, USA, 2008.

[21] B. Scanavino, G. Montorsi, and S. Benedetto, "Convergence properties of iterative decoders working at bit and symbol level," in Proc. IEEE Global Telecommunications Conference, GLOBECOM '01, vol. 2, San Antonio, TX, USA, pp. 1037-1041, 2001.

[22] S. X. Ng, S. Das, J. Wang, and L. Hanzo, "Near-capacity iteratively decoded space-time block coding," in Proc. 67th IEEE Vehicular Technology Conference, VTC '08 Spring, Marina Bay, Singapore, pp. 590594, May 2008.

[23] M. Tüchler, "Convergence prediction for iterative decoding of threefold concatenated systems," in Proc. GLOBECOM '02, Taipei, Taiwan, pp. 1358-1362, Nov. 2002.

[24] F. Brannstrom, L. K. Rasmussen, and A. J. Grant, "Convergence analysis and optimal scheduling for multiple concatenated codes," IEEE Trans. Inf. Theory, vol. 51, pp. 3354-3364, Sept. 2005.

[25] J. Wang, S. X. Ng, L. L. Yang, and L. Hanzo, "Combined serially concatenated codes and MMSE equalization: an EXIT chart aided perspective," in Proc. IEEE 64th Vehicular Technology Conference 2006 (VTC-2006 Fall), pp. 1-5, Sept. 2006.

[26] S. X. Ng, J. Wang, M. Tao, L.-L. Yang, and L. Hanzo, "Iteratively decoded variable-length space-time coded modulation: code construction and convergence analysis," IEEE Trans. Wireless Commun., vol. 6, pp. 1953-1963, May 2007.

[27] J. Hagenauer, "Rate-compatible punctured convolutional codes (RCPC codes) and their applications," IEEE Trans. Commun., vol. 36, pp. 389400, Apr. 1988.

[28] S. Benedetto, D. Divsalar, G. Montorsi, and F. Pollara, "A soft-input soft-output APP module for iterative decoding of concatenated codes," IEEE Commun. Lett., vol. 1, pp. 22-24, Jan. 1997.

[29] S. X. Ng, J. Wang, and L. Hanzo, "Unveiling near-capacity code design: the realization of Shannon's communication theory for MIMO channels," in Proc. IEEE International Conference on Communications (ICC '08), pp. 1415-1419, May 2008.

[30] S. X. Ng, J. Y. Chung, and L. Hanzo, "Turbo-detected unequal protection MPEG-4 wireless video telephony using trellis coded modulation and space-time trellis coding," IEE Proc. Commun., vol. 152, pp. 1116-1124, 2005.

[31] I. Land, P. Hoeher, and S. Gligorević, "Computation of symbol-wise mutual information in transmission systems with $\log$ APP decoders and application to EXIT charts," in Proc. International ITG Conference on Source and Channel Coding (SCC), Erlangen, Germany, pp. 195-202, Jan. 2004.

[32] S. ten Brink, "Convergence behaviour of iteratively decoded parallel concatenated codes," IEEE Trans. Commun., vol. 49, pp. 1727-1737, Oct. 2001.

[33] L. Hanzo, S. X. Ng, T. Keller, and W. Webb, Quadrature Amplitude Modulation: From Basics to Adaptive Trellis-Coded, Turbo-Equalised and Space-Time Coded OFDM, CDMA and MC-CDMA Systems, pp. 746-748. Wiley-IEEE Press, 2nd ed., 2004.

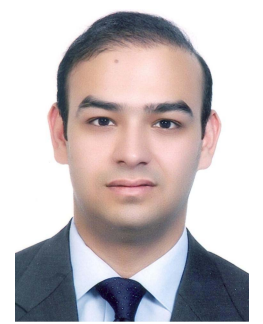

Muhammad Fasih Uddin Butt ( $\left.\mathrm{S}^{\prime} 08\right)$ received his B.E. degree from National University of Sciences \& Technology (NUST), Pakistan in 1999. He received his M.E. degree from Center for Advanced Studies in Engineering, UET Taxila, Pakistan with specialization in Digital Communication/Computer Networks in 2003. He served as faculty member in Department of Electrical Engineering, COMSATS Institute of Information Technology (CIIT), Islamabad, Pakistan from 2002 till 2006. He then won scholarship for Ph.D. studies sponsored by CIIT under the patronage of Higher Education Commission, Government of Pakistan. Since October 2006, he has been working towards his Ph.D. degree at Communications Research Group, School of Electronics and Computer Science, University of Southampton, U.K. His research interests include Channel Coding, EXIT Chart Analysis and Distributed Coding for Cooperative Communications. He is an author of more than ten IEEE publications during his Ph.D. research.

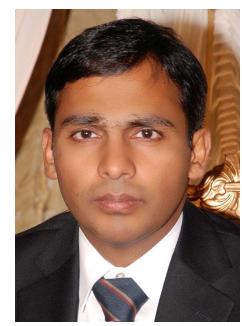

Raja Ali Riaz received his B.Engg. degree from National University of Sciences and Technology (NUST) Pakistan with Silver Medal in 1998. $\mathrm{He}$ is M.Sc Electrical Engineering degree holder from CASE, UET Taxila, Pakistan specialising in Telecommunications in 2003. He has done another M.Sc Electrical Engineering from National University of Sciences and Technology (NUST) Pakistan with controls of dynamic systems as area of specialization. He obtained his Ph.D. degree in the field of ultrawideband communications, School of Electrical and Computer Sciences, University of Southampton, UK in 2010. He is an author of more than ten IEEE publications during his Ph.D. Raja is recipient of foreign Ph.D. Scholarship from COMSATS, HEC, Government of Pakistan. His research interests include Channel Coding, Communication Systems, Stochastic Processes.

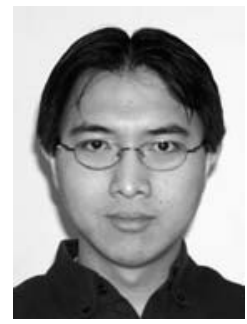

Soon Xin Ng (S'99-M'03-SM'08) received the B.Eng. degree (First class) in electronics engineering and the Ph.D. degree in wireless communications from the University of Southampton, Southampton, U.K., in 1999 and 2002, respectively. From 2003 to 2006, he was a postdoctoral research fellow working on collaborative European research projects known as SCOUT, NEWCOM and PHOENIX. Since August 2006, he has been a lecturer in wireless communications at the University of Southampton. He has been part of a team working on the OPTIMIX European project since March 2008. His research interests are mainly in adaptive coded modulation, channel coding, space-time coding, joint source and channel coding, OFDM, MIMO, cooperative communications and distributed coding. He has published numerous papers and coauthored a book in this field.

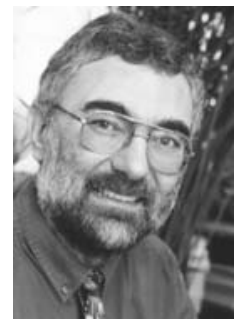

Lajos Hanzo (FREng, FIEEE, FIET, DSc) received his degree in electronics in 1976 and his doctorate in 1983. During his career he has held various research and academic posts in Hungary, Germany and the UK. Since 1986 he has been with the School of Electronics and Computer Science, University of Southampton, UK, where he holds the chair in telecommunications. He has co-authored 19 books on mobile radio communications totalling in excess of 10,000 pages, published 884 research entries at IEEE Xplore, acted as TPC Chair of IEEE conferences, presented keynote lectures and been awarded a number of distinctions. Currently he is directing an academic research team, working on a range of research projects in the field of wireless multimedia communications sponsored by industry, the Engineering and Physical Sciences Research Council (EPSRC) UK, the European IST Programme and the Mobile Virtual Centre of Excellence (VCE), UK. He is an enthusiastic supporter of industrial and academic liaison and he offers a range of industrial courses. He is also an IEEE Distinguished Lecturer as well as a Governor of both the IEEE ComSoc and the VTS. He is the acting Editor-in-Chief of the IEEE Press. For further information on research in progress and associated publications please refer to http://www-mobile.ecs.soton.ac.uk 